\title{
Un programa de rastreo obligatorio con electrocardiograma y ergometría no fue efectivo para reducir la incidencia de muerte súbita en atletas
}

A mandatory electrocardiographic and ergometric test screening program wasn " $t$ effective to reduce the incidence of sudden death in athletes

\section{Objetivos}

Evaluar el impacto de un programa de rastreo de alteraciones cardíacas implementado en Israel desde 1997 sobre la incidencia* de muerte súbita en dicha población.

\section{Diseño, lugar y población}

Búsqueda sistemática realizada entre septiembre y diciembre de 2009 en los dos periódicos principales de Israel (Yediot Ahronot y Maariv, que cubren más del $90 \%$ de lectores) para determinar el reporte de muertes súbitas en atletas entre enero de 1985 y diciembre de 2009. Fueron analizados todos los artículos publicados. Los datos sobre el tamaño de la población en riesgo fueron obtenidos de la Autoridad Deportiva de Israel, aclarando que la Ley de Deportes de Israel definió como atleta a cualquier individuo que participa en actividades deportivas, a cualquier nivel de resistencia física.

\section{Intervención y medición de los resultados principales}

El programa de rastreo incluye la realización anual de un interrogatorio, un examen físico y un electrocardiograma de reposo; y la solicitud de una prueba de esfuerzo a través del protocolo de Bruce (cada cuatro años entre los 17 y 34 años de
Steinvil A y col. J Am Coll Cardiol 2011;57:1291-6. edad y anualmente luego de los 35 ). Ante cualquier hallazgo anormal en dichas pruebas, los pacientes son remitidos al cardiólogo para completar la evaluación.

Se definió muerte súbita a cualquier muerte instantánea, con resucitación no exitosa, vista por testigos, excluyéndose a las relacionadas con traumas.

\section{Resultados principales}

Fueron identificados 24 eventos de muerte súbita durante el periodo analizado. Todos fueron varones, en un rango etario entre 12 a 44 años (media 23,9 +/- 8,8 años). De estos 24 casos, 11 ocurrieron en el periodo anterior a la implementación del programa de rastreo, y 13 posteriormente a la sanción de dicha ley. La incidencia anual de muerte súbita fue de 2,54/100.000 eventos en la etapa previa al rastreo y de $2.66 / 100.00$ durante la etapa posterior $(p=0,88)$.

\section{Conclusiones}

La incidencia de muerte súbita se mantuvo dentro del rango reportado en análisis previos, lo que sugiere que este programa de rastreo no aporta mayores beneficios.

\section{Comentario}

Es razonable preguntarse si la MS, mas allá del componente trágico que indudablemente conlleva, cumple con los requisitos para ser rastreada poblacionalmente. Dos criterios son particularmente importantes: 1) conocer si esta condición constituye un problema de salud pública, lo que depende no solo de su gravedad (que está fuera de discusión) sino también de cuan frecuentemente ocurre en la población general y de que probabilidades hay de reducir los daños asociados a la misma; 2) que la prueba de rastreo a realizar posea una sensibilidad y especificidad adecuadas para detectar la enfermedad en estadios tempranos.

En relación al primer ítem, tanto en este estudio como en publicaciones anteriores ${ }^{1}$, podemos observar que la frecuencia de este evento es baja: 2,6 /100.000 eventos al año según el estudio israelí, 3,6/100.000 en un estudio realizado en Italia en 2006 (el único estudio que había informado beneficios asociados a un programa de rastreo) y 1/100.000 en EE.UU. durante 2009.

En cuanto a las características operativas de la prueba de detección, se sabe que la sensibilidad del ECG para detectar enfermedades cardiacas en población asintomática es cercana al $50 \%$, con gran variabilidad en diferentes condiciones (miocardiopatías, enfermedad coronaria, arritmias, etc.). Otro factor importante a tener en cuenta es su baja especificidad (60\%), lo que implica una incidencia de $40 \%$ de resultados falsamente positivos en la población sana, con la consiguiente repercusión emocional, estigmatización y exclusión innecesaria de muchos individuos de algunas actividades deportivas. Mas allá de estas consideraciones, un trabajo recientemente publicado por
Magalski y col. ${ }^{2}$ demostró que el agregado de un ECG a una evaluación precompetitiva en 964 atletas universitarios de EE.UU. permitió identificar a nueve con alteraciones cardíacas potencialmente serias y a excluir de la competencia a dos de ellos.

Respecto del trabajo que hemos resumido, destacamos varias debilidades metodológicas: a) a diferencia del masivo programa italiano de rastreo que es para todos los deportistas, el israelí sólo está destinado a los de alto rendimiento, lo que es un problema a la hora de sacar conclusiones ya que el $90 \%$ de las MS ocurre en deportistas recreativos ${ }^{3}$ y tienen bajísima probabilidad de ser publicadas en los periódicos; b) al no haber sido un trabajo prospectivo, carga con el sesgo de registro de todo trabajo retrospectivo, que puede conducir a dejar de lado algunas muertes que habrían merecido ser incluidas en el análisis (vale destacar que durante dicho período ocurrieron muertes durante el entrenamiento del ejército Israelí que no fueron tenidas en cuenta).

\section{Conclusiones de los comentadores}

Todavía quedan interrogantes sin responder respecto de cuál es la mejor estrategia de rastreo de potenciales predisponentes a la MS en atletas.

Nota: para complementar este tema, se remite al lector a dos actualizaciones afines sobre evaluación cardiovascular y muerte súbita en deportistas, publicadas ambas en este número de EVIDENCIA.

Yamila Mahumud [ Servicio de Medicina Familiar y Comunitaria del Hospital Italiano de Buenos Aires. yamila.mahumud@hospitalitaliano.org.ar ] Diego Iglesias [ Servicio de Cardiología del Hospital Italiano de Buenos Aires diego.iglesias@hospitalitaliano.org.ar ]

Recibido el 15/06/11 y aceptado el 05/09/11

Mahumud $Y$ e Iglesias D. Un programa de rastreo obligatorio con electrocardiograma y ergometría no fue efectivo para reducir la incidencia de muerte súbita en atletas. Evid Act Pract Ambul. Jul-Sep 2011;14(3): 89. Comentado de: Steinvil A y col. Mandatory electrocardiographic screening of athlets to reduce their risk for sudden death. J Am Coll Cardiol 2011:57:1291-6.

\section{Referencias}

1 Maron B y col. Comparison of U.S. and Italian experiences with sudden cardiac deaths in young competitive athletes and implications for preparticipation screening strategies. Am J Cardiol 2009;104:276-80

2. Magalski A y col. Cardiovascular Screening with Electrocardiography and Echocardiography in Collegiate Athletes. The American Journal of Medicine (2011) 124, 511-518.

3. Marijon E y col. Sports-Related Sudden Death in the General Population. Circulation. 2011;124:672-681. 